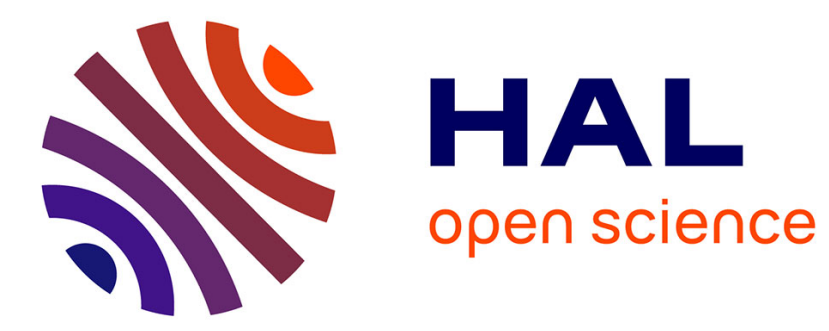

\title{
Influence of energetics on the thresholds of heavy ion transfer reactions
}

\author{
D. Gardes, R. Bimbot, Agnès Fleury, F. Hubert, M.F. Rivet
}

\section{To cite this version:}

D. Gardes, R. Bimbot, Agnès Fleury, F. Hubert, M.F. Rivet. Influence of energetics on the thresholds of heavy ion transfer reactions. Journal de Physique Lettres, 1975, 36 (3), pp.59-63. 10.1051/jphyslet:0197500360305900 . jpa-00231154

\section{HAL Id: jpa-00231154 https://hal.science/jpa-00231154}

Submitted on 1 Jan 1975

HAL is a multi-disciplinary open access archive for the deposit and dissemination of scientific research documents, whether they are published or not. The documents may come from teaching and research institutions in France or abroad, or from public or private research centers.
L'archive ouverte pluridisciplinaire HAL, est destinée au dépôt et à la diffusion de documents scientifiques de niveau recherche, publiés ou non, émanant des établissements d'enseignement et de recherche français ou étrangers, des laboratoires publics ou privés. 
Classification

Physics Abstracts

4.375

\title{
INFLUENCE OF ENERGETICS ON THE THRESHOLDS OF HEAVY ION TRANSFER REACTIONS
}

\author{
D. GARDÈS, R. BIMBOT, A. FLEURY (*), F. HUBERT $\left(^{*}\right)$ and M. F. RIVET
}

Laboratoire de Chimie Nucléaire, Institut de Physique Nucléaire, B.P. 1, 91406 Orsay, France

(Reçu le 14 novembre 1974, accepté le 9 janvier 1975)

\begin{abstract}
Résumé. - La possibilité de transferts sous-coulombiens est discutée du point de vue de l'énergétique de la réaction. La position relative des courbes d'énergie potentielle pour les voies d'entrée et de sortie, corrigées par la perte de masse $Q_{\mathbf{g g}}$ de la réaction étudiée, joue un rôle déterminant. Lorsque le potentiel $V_{\mathrm{i}}(R)$ de la voie d'entrée est située au-dessus de celui de la voie de sortie $V_{f}(R)-Q_{g g}$, le transfert est énergétiquement possible ; il intervient dès que les noyaux sont suffisamment proches l'un de l'autre et cela même pour des énergies inférieures à la barrière. Une comparaison est faite avec des résultats expérimentaux.
\end{abstract}

\begin{abstract}
The possibility of sub-barrier transfers is discussed from the point of view of the energetics of the reaction. The position of the entrance channel potential energy curve $V_{\mathrm{i}}(R)$ relative to that of the exit channel corrected for the mass balance $V_{\mathrm{f}}(R)-Q_{\mathrm{gg}}$ appears to be crucial. When the former curve is situated above the latter one, the transfer is energetically probable and will occur as soon as the colliding nuclei are sufficiently close to each other, i.e. for sub-barrier incident energies. Experimental examples are given.
\end{abstract}

Considerable experimental and theoretical attention has recently been centered on questions that relate to thresholds of nuclear reactions and to the interaction barrier [1-5]. However, the notion of interaction barrier is still not quite clear. Generally, the interaction barrier is defined as the value $B_{\mathrm{i}}$ at which the interaction potential (nucleon + Coulomb) for the s-wave passes through a maximum; but this must be regarded as a strong interaction barrier, or a fusion barrier and some nuclear reactions, namely transfer reactions can take place at incident energies somewhat lower than $B_{\mathrm{i}}$. The above definition, however, will be adopted here, and in considering the possibility of sub-barrier reactions, we shall ask the following question : for a given projectile target system, can one predict whether or not a given transfer reaction will occur with significant cross section for incident energies lower than $B_{\mathrm{i}}$ ? This question will be studied here from the point of view of the energetics involved in the reaction, which have been shown, in previous studies [6-13], to play a fundamental role. Many authors have underlined the influence of the $Q$ value on reaction probabilities,

(*) Laboratoire de Chimie Nucléaire, Centre d'Etudes Nucléaires de Bordeaux, 33170 Gradignan, France. and the enhancements of transitions for which $Q=Q_{\text {opt }}$, where

$$
Q_{\mathrm{opt}}=E_{\mathrm{i}}\left[\frac{Z_{3} Z_{4}}{Z_{1} Z_{2}}-1\right]
$$

$E_{\mathrm{i}}$ being the c.m. incident energy, $Z_{1} Z_{2}$ the atomic numbers of the colliding nuclei and $Z_{3} Z_{4}$ those of the transfer products [6-9]. Others [10-11] have discussed the dramatic influence on the transfer cross sections of $Q_{\mathrm{gg}}-\Delta V_{\mathrm{c}}, Q_{\mathrm{gg}}$ being the $Q$ value for a ground state transition, and $\Delta V_{c}$ the difference between the Coulomb energies in the final and initial states $\Delta V_{\mathrm{c}}=V_{\mathrm{cf}}-V_{\mathrm{ci}}$.

Similar ideas are involved in the present paper, but firstly we shall focus on the feasibility of subbarrier reactions, and secondly we shall include a nuclear part in addition to $V_{\mathrm{c}}$ in the expression for the interaction potential. This nuclear contribution may have a non negligible influence for incident energies slightly lower than the interaction barrier.

1. Experimental evidence. - Let us consider the two systems ${ }^{14} \mathrm{~N}+{ }^{209} \mathrm{Bi}$ and ${ }^{16} \mathrm{O}+{ }^{209} \mathrm{Bi}$. In figures 1 and 2 are shown the experimental excitation functions obtained by activation techniques $[14,15]$ for ${ }^{210} \mathrm{Po}$ and ${ }^{211} \mathrm{At}$ which correspond respectively to transfers of 1 and 2 protons from the projectile 
to the target. The excitation function for ${ }^{211} \mathrm{Rn}$ production is also plotted in figure 1. From angular distributions and recoil range measurements, it was established in reference [14] that this production is made through the compound nucleus reaction : ${ }^{14} \mathrm{~N}+{ }^{209} \mathrm{Bi} \rightarrow{ }^{219} \mathrm{Th}+4 \mathrm{n}$ followed by two fast $\alpha$ decays. Therefore, in order to induce this reaction the projectile must overcome the strong interaction barrier. The vertical solid line indicates the value of the interaction barrier calculated with a WoodsSaxon nuclear potential (see below).

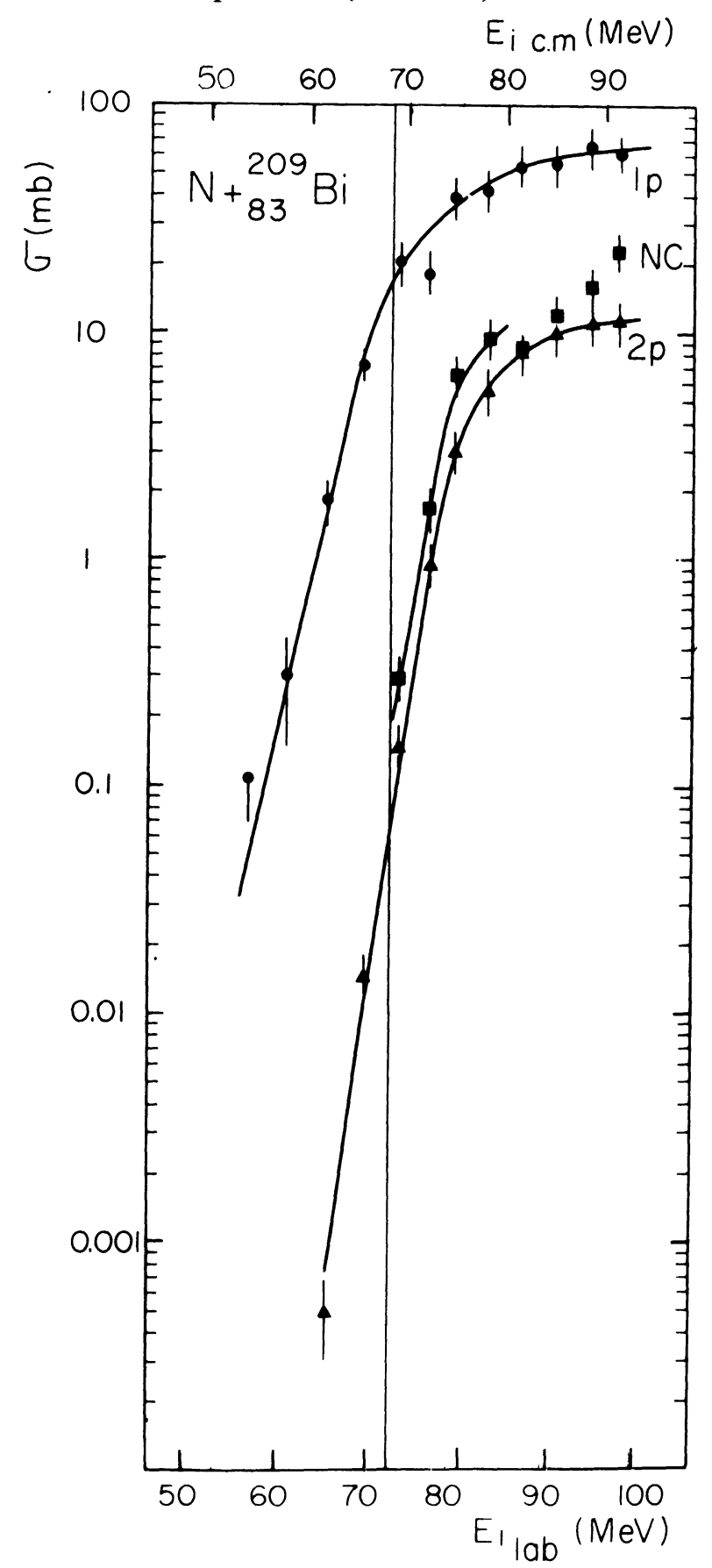

FIG. 1. - Excitation functions for the reactions ${ }^{14} \mathrm{~N}+{ }^{209} \mathrm{Bi} \rightarrow{ }^{210} \mathrm{Po}$ $(\phi),{ }^{211} \mathrm{At}(\phi)$ and ${ }^{211} \mathrm{Rn}(\boldsymbol{)})$, corresponding respectively to $1 \mathrm{p}$-transfer, $2 \mathrm{p}$-transfer, and a compound nucleus process. The lines are drawn to guide the eye. The vertical line indicates the interaction barrier. The data are from reference [14].
It can be seen in figures 1 and 2 that the relative positions of single-proton and two-proton transfer excitation functions for the two systems are quite different. With a nitrogen beam, the $1 \mathrm{p}$-transfer reaction has a significant cross section for energies below the interaction barrier. This is not the case for the 2p-transfer cross section. On the contrary, with the oxygen beam, both excitation functions rise somewhat below the interaction barrier.

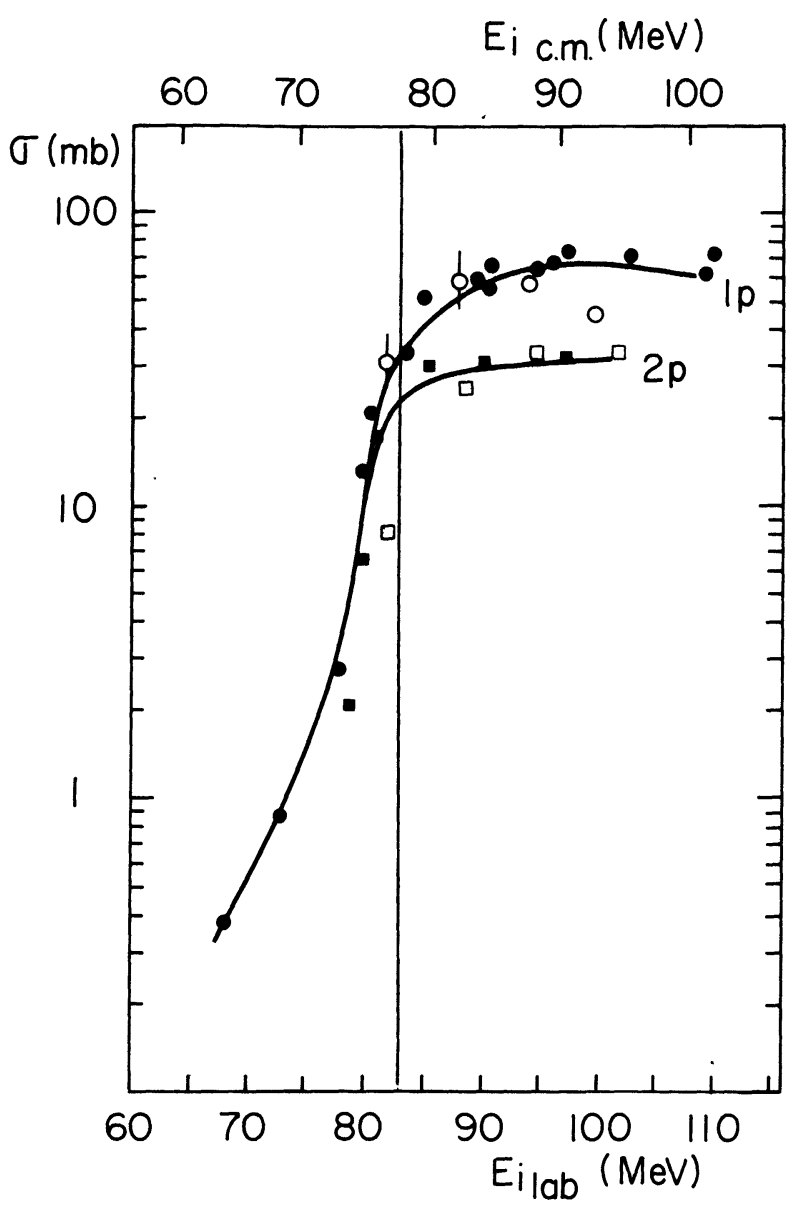

FIG. 2. - Excitation functions for the reactions ${ }^{16} \mathrm{O}+{ }^{209} \mathrm{Bi} \rightarrow{ }^{210} \mathrm{Po}$ (circles) and ${ }^{211} \mathrm{At}$ (squares). Open symbols are data from reference [14], black symbols are from reference [15]. The lines are drawn to guide the eye. The vertical line indicates the interaction barrier.

2. Potential energy curves. - In order to explain such behaviour, one must consider the energetics of the particular reactions : the incident c.m. energy $E_{\mathrm{i}}$ can be divided into a potential energy $V_{\mathrm{i}}(R)$ and a kinetic energy $E_{\mathrm{ki}}(R)$, according to the distance $R$ between the centers of the nuclei. The interaction potential $V_{\mathrm{i}}(R)$ is the sum of a repulsive Coulomb part $V_{\mathrm{ci}}(R)$ and an attractive nuclear part $V_{\mathrm{Ni}}(R)$. Only head-on collisions $(l=0)$ will be considered in this paper which deals with reactions induced near or below the barrier. The centrifugal part $V_{\text {ce }}(l, R)$ of the interaction potential will therefore be ignored. 
The general shape of the curve $V_{\mathrm{i}}(R)$ is shown in figure 3 . Its maximum defines the value of the interaction barrier $B_{\mathbf{i}}$.

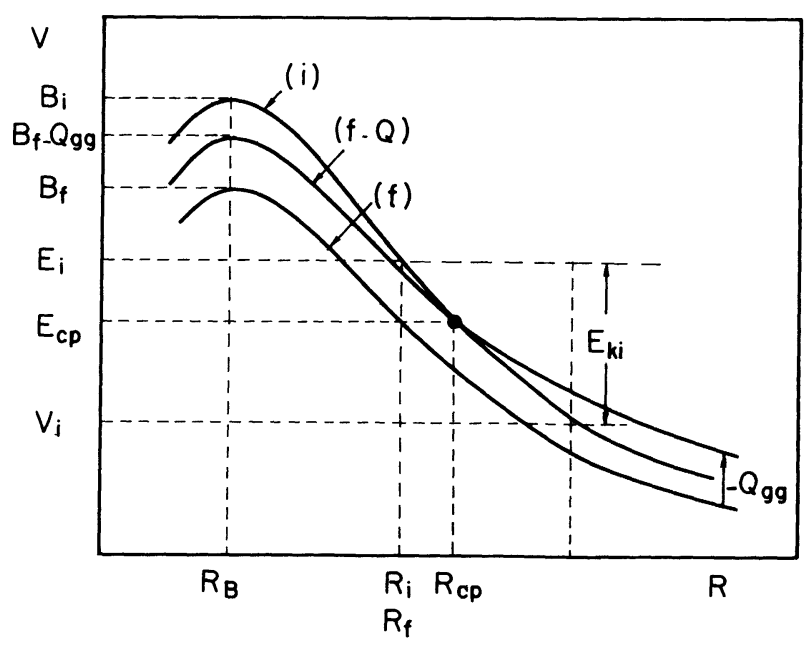

FIG. 3. - Interaction potentials in the entrance and exit channels (see text).

If $E_{\mathrm{i}}<B_{\mathrm{i}}$, the two nuclei will not be able to come closer than a certain distance $R_{\mathrm{i}}$ at which $E_{\mathrm{ki}}=0$. The transfer reaction will occur with a significant probability if two conditions are fulfilled :

a) Proximity condition : the nuclei must be close enough to each other to have the possibility of exchanging matter.

b) Energy condition : the reaction must be energetically probable. As will be shown in what follows, this second condition is directly related to the relative position of the interaction potentials in the entrance and exit channels.

For the purpose of simplicity, it is assumed that the transfer occurs only at the closest distance of approach $R_{\mathrm{i}}$, the nuclei being at rest in the c.m. system $\left(E_{\mathrm{ki}}=0\right)$. The distance $R_{\mathrm{f}}$ between the centers of the nuclei after transfer is assumed to be equal to $R_{\mathrm{i}}$, and the kinetic energy $E_{\mathrm{kf}}$ equal to zero. These assumptions neglect recoil and quantum effects.

The energy balance can then be written as

$$
V_{\mathrm{i}}\left(R_{\mathrm{i}}\right)+Q_{\mathrm{gg}}=V_{\mathrm{f}}\left(R_{\mathrm{i}}\right)+E_{\mathrm{f}}^{*}
$$

where $V_{\mathrm{i}}$ and $V_{\mathrm{f}}$ are the interaction potentials in the entrance and exit channels, and $E_{\mathrm{f}}^{*}$ the excitation energy of the final nuclei.

The reaction will be energetically possible if $E_{\mathrm{f}}^{*} \geqslant 0$. This condition is equivalent to

$$
V_{\mathrm{i}} \geqslant V_{\mathrm{f}}-Q_{\mathrm{gg}}
$$

and the solution of this inequality can be obtained by comparing the curves $V_{\mathrm{i}}(R)$, (denoted by $(i)$ in figure 3) and $V_{\mathrm{f}}(R)-Q_{\mathrm{gg}}$ denoted by $(f-Q)$. For example, if charge is transferred from projectile to target, the curve $V_{\mathrm{f}}(R)$ is always situated below the curve $V_{\mathrm{i}}(R)$. If $Q_{\mathrm{gg}}$ is negative, the relative positions of the relevant curves are as given in figure 3 .
The existence of a crossing point for the curves ( $i$ ) and $(f-Q)$ can be discussed in terms of the values of $Q_{\mathrm{gg}}$ and $\Delta B=B_{\mathrm{f}}-B_{\mathrm{i}}$ (see Fig. 3).

- If $B_{\mathrm{i}}>B_{\mathrm{f}}-Q_{\mathrm{gg}}$, i.e.

$$
Q_{\mathrm{gg}}-\Delta B>0 \text {, }
$$

a crossing point will exist at a distance of approach $R_{\mathrm{cp}}$ which is larger than the distance corresponding to the interaction barrier, $R_{\mathrm{B}}$. For $R<R_{\mathrm{cp}}$, condition (3) will be satisfied. Therefore, the energy $E_{\mathrm{cp}}=V_{\mathrm{i}}\left(R_{\mathrm{cp}}\right)$ can be considered as the energetic threshold for the transfer reaction and for incident energies higher than $E_{\mathrm{cp}}$, the transfer will be energetically favoured. However, the reaction will be effectively observed only if the proximity condition (a) is also fulfilled. Therefore, $E_{\mathrm{cp}}$ must be taken as a lower limit for the reaction threshold (see the restrictions of remark 1 below). If the value of $E_{\mathrm{cp}}$ is much lower than $B$, the reaction will be produced as soon as condition (a) is satisfied, and significant sub-barrier cross sections will be observed.

- If $Q_{\mathrm{gg}}-\Delta B<0$, no crossing point exists for $R>R_{\mathrm{B}}$ and the transfer reaction will not have a significant cross section below the barrier.

Two remarks can be made about the previous statement.

Remark 1. - Because of the assumptions neglecting recoil and quantum effects, the value $E_{\text {cp }}$ defined above must not be taken as an effective threshold under which the reaction cannot occur : the reaction can be induced for energies slightly lower than $E_{\mathrm{cp}}$, if for example $E_{\mathbf{k i}} \neq 0$ or if $R_{\mathrm{f}} \neq R_{\mathrm{i}}$. But the incident energy $E_{\mathrm{i}}=E_{\mathrm{cp}}$ should correspond to a sharp increase in the cross section.

Remark 2. - If one neglects the nuclear part of the interaction potential, the quantity $Q_{\mathrm{gg}}-\Delta B$ becomes $Q_{\mathrm{gg}}-\Delta E_{\mathrm{c}}$, and one can understand the relevance of this quantity as far as transfer probabilities are concerned [10-11].

Moreover, condition (3) then becomes

$$
\frac{Z_{1} Z_{2} e^{2}}{R} \geqslant \frac{Z_{3} Z_{4} e^{2}}{R}-Q_{\mathrm{gg}} .
$$

This condition will be fulfilled at the minimum distance of approach $R_{\mathrm{i}}$ for incident energies

$$
E_{\mathrm{i}}=Z_{1} Z_{2} e^{2} / R_{\mathrm{i}}
$$

such that

$$
E_{\mathrm{i}}-\frac{Z_{3} Z_{4} e^{2}}{R_{\mathrm{i}}} \geqslant-Q_{\mathrm{gg}} .
$$

This inequality is equivalent to $Q_{\mathrm{opt}} \leqslant Q_{\mathrm{gg}}$ (see eq. (1)).

Therefore, an approximate value $E_{\mathrm{cp}}^{\prime}$ of the energy corresponding to the crossing point can be found by solving the following equation :

$$
E_{\mathrm{cp}}^{\prime}=\frac{Q_{\mathrm{gg}}}{Z_{3} Z_{4} / Z_{1} Z_{2}-1}
$$


Refinements can be made to take into account recoil effects in the expression for $Q_{\text {opt }}$.

3. Application to experimental data. - The potential energy curves have been drawn for the transfer reactions mentioned in section 1 . The following expressions were used for the entrance channel

$$
V_{\mathrm{ci}}(R)=\frac{Z_{1} Z_{2} e^{2}}{R},
$$

where $Z_{1}$ and $Z_{2}$ denotes atomic numbers of projectile and target and

$$
V_{\mathrm{Ni}}(R)=V_{0}\left[1+\exp \left(\frac{R-R_{\mathrm{T}}}{d}\right)\right]^{-1}
$$

(Woods-Saxon form) with $V_{0}$ (depth parameter) $=-50 \mathrm{MeV}, d$ (diffuseness parameter) $=0.6 \mathrm{fm}$, and $R_{\mathrm{T}}=r_{0}\left(A_{1}^{1 / 3}+A_{2}^{1 / 3}\right) A_{1}$ and $A_{2}$ being respectively the atomic masses of projectile and target, and $r_{0}=1.22 \mathrm{fm}$.

Such an expression for the interaction potential gives a correct description of the outer surface of the nucleus which is of interest here [16]. The values adopted for the nuclear parameters are not critical, and the conclusions which follow are not affected by small changes in these values.

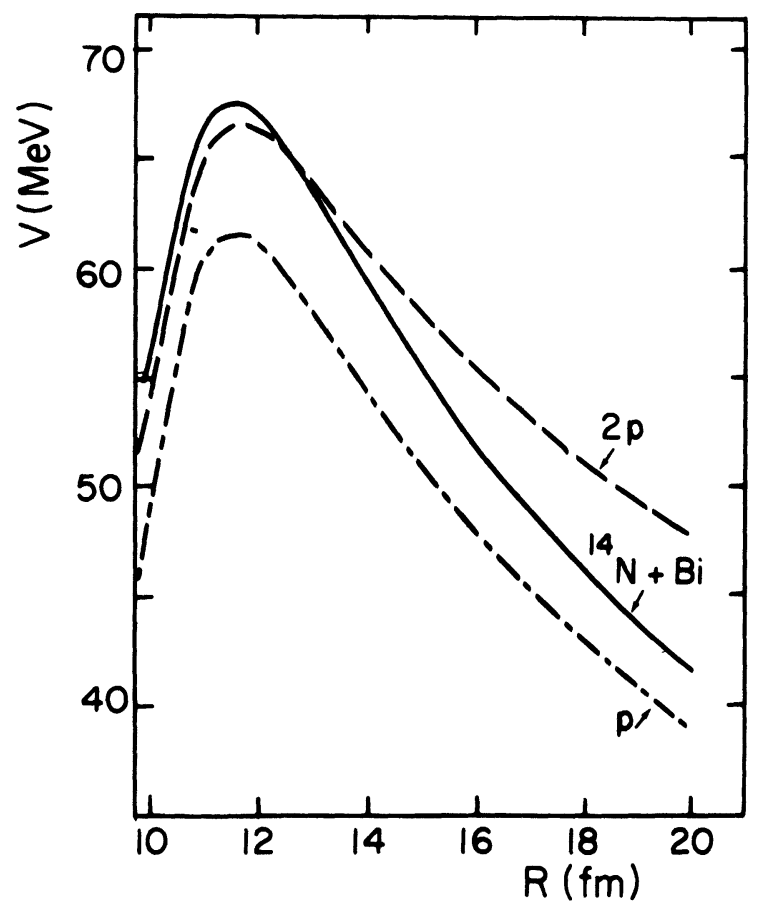

Fig. 4. - Potential energy curves involved in the transfers of one and two protons induced with ${ }^{14} \mathrm{~N}$ on ${ }^{209} \mathrm{Bi}$. The interaction potential $V_{\mathrm{i}}$ is plotted versus the distance of approach for the entrance channel, and the quantities $V_{\mathrm{f}}-Q_{\mathrm{gg}}$ are plotted for the exit channels.

As far as the exit channel is concerned, the quantity $V_{\mathrm{f}}(R)-Q_{\mathrm{gg}}$ was calculated with similar expressions for $V_{\mathrm{cf}}(R)$ and $V_{\mathrm{Nf}}(R)$ to those given above.
It can be seen in figure 4 that the transfer of one proton in the case of ${ }^{14} \mathrm{~N}$ on ${ }^{209} \mathrm{Bi}$ is energetically favoured even for very low incident energies

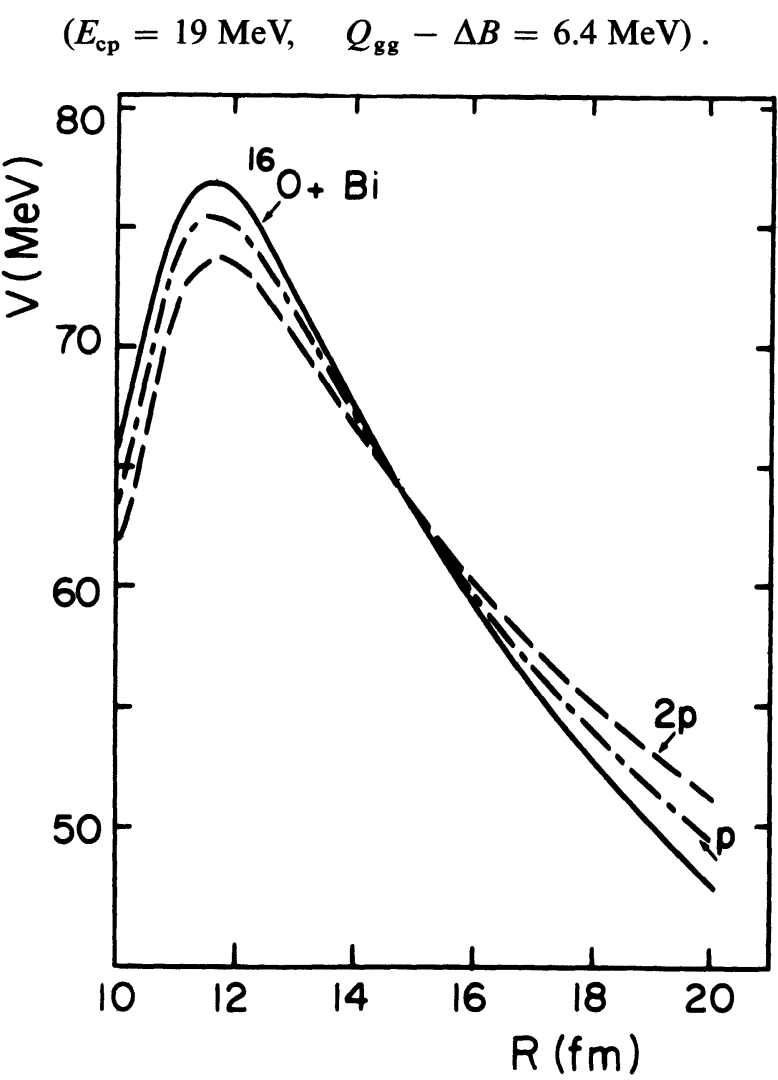

FIG. 5. - Same as figure 4 but for transfer reactions induced with ${ }^{16} \mathrm{O}$ on ${ }^{209} \mathrm{Bi}$.

The only limitation to proton transfer is therefore the proximity condition. This case thus involves high cross sections below the barrier, as can be seen in figure 1. In contrast, the energetic conditions are much more critical for the $2 \mathrm{p}$-transfer reaction : $\left(E_{\mathrm{cp}}=65 \mathrm{MeV}, Q_{\mathrm{gg}}-\Delta B=1.2 \mathrm{MeV}\right)$; the crossing occurs only close to the barrier $B_{\mathrm{i}}=68 \mathrm{MeV}$. This restriction is well reflected in experimental data (Fig. 1).

For the projectile ${ }^{16} \mathrm{O}$, the behaviours of the potential energy curves corresponding to one- and twoproton transfers are very similar to each other (see Fig. 1). The barrier $B_{\mathrm{i}}$ is at $77 \mathrm{MeV}$, the crossings are observed at $E_{\mathrm{cp}} \simeq 64 \mathrm{MeV}$ for both reactions, and the values of $Q_{\mathrm{gg}}-\Delta B$ are respectively $2 \mathrm{MeV}$ (1p-transfer) and $3.6 \mathrm{MeV}$ (2p-transfer). This is in agreement with the similarities observed in the shapes and positions of the experimental excitation functions (Fig. 2).

In conclusion, we note that from the data discussed here, the comparison of potential energy curves appears to be a good guide in predicting the feasibility of sub-barrier transfer reactions.

We are very grateful to $M$. Lefort for helpful discussions, and to F. Plasil for a careful reading of the manuscript. 


\section{References}

[1] Wond, C. Y., Phys. Lett. 42B (1972) 186; Phys. Lett. 31 (1973) 766

[2] Lefort, M., Le Beyec, Y., Pétbr, J., Riv. Nuov. Cim. 4 (1974) 79-98.

[3] Noô, C., Tamain, B., Galin, J., Beiner, M., Lombard, R. J., IPNO-TH-74.19 (submitted to Nucl. Phys.).

[4] Vaz, L. C. and Alexander, J. M., Phys. Rev. 10 (1974) 464.

[5] BlanN, M., Proc. Int. Conf. Nucl. Phys., Munich 1973, J. de Boer et H. J. Mang, Edit. Vol. 2 (1973) 658.

[6] Butten, P. J. A. and Goldfarb, L. J. B., Nucl. Phys. A 176 (1971) 299.

[7] BRINK, D. M., Phys. Lett. 40B (1972) 37.

[8] Von Oertzen, W., Bohlen, H. G. and Gebauer, B., Nucl. Phys. A 207 (1973) 91.

[9] Schiffer, J. P., KÖRNER, H. J., Sibmssen, R. H., Jones, K. W. and Schwarzschild, A., Phys. Lett. 44B (1973) 47.
[10] Diamond, R. M., Poskanzer, A. M., Stephens, F. S., SwiaTECKI, W. J. and WARD, D., Phys. Rev. Lett. 20 (1968) 802.

[11] Galin, J., GatTy, B., Lefort, M., PÉter, J. and Tarrago, X., Phys. Rev. 182 (1969) 1267.

[12] Artukh, A. G., Avdeichikov, V. V., ERö, J., Gridnev, G. F., MikHeEv, V. L., Volkov, V. V. and WILCZYNSKI, J., Nucl. Phys. A 160 (1971) 511.

[13] Siemens, P. J., Bondorf, J. P., Gross, D. M. E. and DickMANN, F., Phys. Lett. 36B (1971) 24.

[14] Gardès, D., Bimbot, R., Maison, J. and Rivet, M. F. (to be published).

[15] Croft, P. D., Alexander, J. M. and Street, K., Phys. Rev. 165 (1968) 1380.

[16] Da Silveira, R., Phys. Lett. 50B (1974) 237. 